\title{
Estudio de la cinética de adsorción de Cu y Hg a partir de soluciones binarias usando quitosano
}

\author{
I. Matus, L. Paniagua y M. Benavente* \\ Facultad de Ingeniería Química, Universidad Nacional de Ingeniería (UNI) \\ Avenida Universitaria, PO Box 5595, Managua, Nicaragua \\ E-mail: bena@kth.se
}

(recibido/received: 28-Enero-2011; aceptado/accepted: 3-Junio-2011)

\begin{abstract}
RESUMEN
En este trabajo se estudió la cinética de adsorción de $\mathrm{Cu}(\mathrm{II})$ y $\mathrm{Hg}(\mathrm{II})$ en quitosano a partir de una solución binaria, a diferentes concentraciones iniciales de los iones metálicos, tamaño de partículas del adsorbente y velocidad de agitación. El quitosano fue extraído por métodos químicos de caparazón de camarón. Soluciones de 1 L de Cu(II) y $\mathrm{Hg}$ (II) fueron puestas en contacto con $2 \mathrm{~g}$ de quitosano y se agitaron por un período de $8 \mathrm{~h}$. Al final del proceso, las muestras fueron analizadas en un espectrómetro de absorción atómica. Los datos experimentales fueron evaluados aplicando los modelos cinéticos de pseudo-primer orden y pseudo-segundo orden. Los resultados del proceso de adsorción de los iones metálicos Cu(II) y Hg(II) con quitosano, mostraron que en la mayoría de los casos, ambos iones fueron eficazmente adsorbidos por la quitosano, obteniéndose un porcentaje de adsorción entre 44-100 \% para $\mathrm{Cu}(\mathrm{II})$ y entre 95-100 \% para el Hg(II). Para ambos iones, los resultados mostraron que a bajas concentraciones el proceso de adsorción se ajusta mejor al modelo de pseudo-primer orden y a concentraciones altas el modelo que mejor se ajusta a los datos experimentales es el modelo de pseudo-segundo orden; es decir, el paso limitante en la velocidad es la reacción de adsorción y no la transferencia de masa.
\end{abstract}

Palabras claves: Caparazón de camarón; Cinética de adsorción; Modelos cinéticos; Solución binaria; Quitosano

\begin{abstract}
In this work, the adsorption kinetic of $\mathrm{Cu}(\mathrm{II})$ and $\mathrm{Hg}(\mathrm{II})$ onto chitosan was studied from a binary solution at different initial concentration of metallic ions, at different particle sizes and agitation rate. Chitosan was obtained from shrimp shells. Solutions of $1 \mathrm{~L}$ of $\mathrm{Cu}(\mathrm{II})$ and $\mathrm{Hg}$ (II) were placed in contact with $2 \mathrm{~g}$ of chitosan and the mixture was agitated by $8 \mathrm{~h}$. Afterwards, the samples were analysed by atomic absorption. The experimental data were tested by application of kinetic models of pseudo-first order and pseudo-second order. The results of adsorption process showed that in the most cases, both ions were effectively adsorbed by chitosan. The percentage of adsorption was 43-100 \% and 94-100 \% for $\mathrm{Cu(II)} \mathrm{and} \mathrm{Hg}(\mathrm{II})$, respectively. The results also showed that at low concentrations, experimental data were better adjusted by pseudo-first order models; meanwhile, at high concentration, the data were well described by the pseudo-second model. Therefore, the rate-limiting step is the adsorption reaction and not mass transfer.
\end{abstract}

Keywords: Adsorption kinetics; Binary solution; Chitosan; Kinetic models; Shrimp shell

*Autor para la correspondencia 


\section{INTRODUCCIÓN}

Nicaragua posee una gran variedad de recursos hídricos (aguas superficiales y subterráneas), muchos de los cuales están siendo contaminados de diferentes maneras, ya sea por la deposición directa de efluentes industriales, efluentes domésticos, desechos agrícolas y desechos mineros (el cual es el mayor proveedor de metales pesados a las aguas) o por contaminación natural. Esta última se presenta principalmente en el occidente del país (Benavente, 2008).

Debido a la importancia de los ecosistemas acuáticos para los seres vivos es primordial reducir las concentraciones de metales pesados. Para ello, se han utilizado diversos métodos para la remoción de éstos contaminantes, tales como coagulación, intercambio iónico, osmosis inversa y precipitación. Sin embargo, la aplicación de estos métodos a veces resulta muy costosa y en algunos casos, no logran alcanzar los límites establecidos por las normas reguladoras de la calidad de agua del país (Benavente, 2008). Como una alternativa ante esta problemática y además, económica, es la utilización de quitosano, un polímero natural que se extrae de los desechos de crustáceos, hongos e insectos (Muzzarelli, 1977).

El quitosano es un polisacárido que presenta un gran poder de adsorción y es capaz de fijar una gran variedad de metales (Muzzarelli, 1977). Entre sus características principales se encuentran: inocuidad a la salud humana, ser el único polielectrólito catiónico natural, poder filmogénico y biodegradable. En la actualidad, el quitosano y sus derivados presentan numerosas aplicaciones en distintas áreas, principalmente en medicina, farmacia, tratamiento de aguas naturales y efluentes, cosmética y medio ambiente (Pastor de Abram, 2004).

Desde 2005, en la Universidad Nacional de Ingeniería (UN) se han desarrollado diferentes estudios dirigidos a la adsorción de iones metálicos con quitosano ( $\mathrm{Fe}, \mathrm{Cu}, \mathrm{Zn}, \mathrm{Hg}$ y $\mathrm{Pb}$ ). En estos trabajos se comprobó que la capacidad de adsorción de estos iones depende del pH de la solución y que la máxima capacidad de adsorción ocurre a pH 6 (Álvarez, 2007). Así mismo, se encontró que en el rango de trabajo, la capacidad de adsorción sigue la secuencia $\mathrm{Hg}>$ $\mathrm{Cu}>\mathrm{Zn}>\mathrm{As}$, y que la cinética de adsorción de los diferentes metales es mejor descrita bajo el modelo de pseudo-segundo orden (Benavente, 2008).

Sin embargo, estos estudios se han basado principalmente en sistemas acuosos conteniendo únicamente un ión metálico. Para la aplicación del quitosano en el tratamiento de aguas naturales y/o aguas residuales en sistemas reales se hace necesario encontrar información adicional acerca de la interacción entre los iones metálicos y el adsorbente; y la influencia que puede tener la presencia de otro ión metálico en la capacidad de adsorción.

El objetivo principal de este trabajo fue analizar la cinética en la capacidad de adsorción del quitosano cuando se tienen dos iones metálicos presentes en una solución variando algunos parámetros tales como concentración inicial de los iones metálicos, velocidad de agitación y tamaño de partícula del adsorbente. Este conocimiento podrá ser utilizado en la aplicación del quitosano como un filtro de intercambio iónico en sistemas de tratamiento de aguas naturales y residuales, y de esta forma contribuir a la disminución de la contaminación de los ecosistemas acuáticos.

\section{METODOLOGÍA}

\section{Material}

a) Quitina

La quitina que se utilizó para obtener quitosano fue facilitada por Hernández y Escorcia (2009). Este material fue extraído de caparazón de camarón proporcionado por la empresa Camarones de Nicaragua, S.A. (CAMANICA).

b) Soluciones de iones metálicos

Las soluciones de los iones metálicos a diferentes concentraciones (ver Tabla 1) fueron preparadas a partir de soluciones estándares de 
$1000 \mathrm{mg} / \mathrm{L}$ de Cobre (II) (marca Merck) y de $1000 \mathrm{mg} / \mathrm{L}$ de Mercurio (II) (marca Fischer). Se consideró trabajar a bajas concentraciones iniciales para $\mathrm{Hg}$ (II) debido a que este ión no se encuentra en grandes cantidades en la naturaleza (Benavente et al., 2007).

\section{Método}

a) Obtención de quitosano

El proceso constó de cuatro etapas: desacetilación, lavado, secado y trituración del producto. En la etapa de desacetilación, se agregaron $750 \mathrm{~mL}$ de $\mathrm{NaOH}$ al $50 \%$ (grado comercial) a $150 \mathrm{~g}$ de quitina contenida en un vaso de precipitado de $2 \mathrm{~L}$. La mezcla se introdujo en el horno a una temperatura de 100 ${ }^{\circ} \mathrm{C}$ por $1 \mathrm{~h}$ y posteriormente, el producto se lavó con agua destilada hasta que la solución de lavado alcanzó un pH de aproximadamente 7. El quitosano producido se filtró y se secó.

Para obtener diferentes tamaño de partícula del adsorbente, el material se trituró en un procesador de alimentos marca Oster y se hizo pasar por tamices de diferente diámetro: $<0.22$ $\mathrm{mm}$ y entre $0.22-0.45 \mathrm{~mm}$ (Fig. 1).

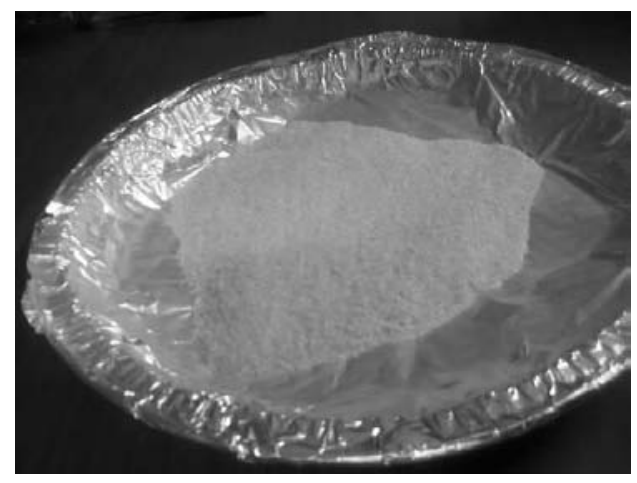

Fig. 1 Quitosano producida a nivel de laboratorio a partir de desechos de camarón.

Posteriormente, para evaluar el grado de desacetilación (DD) del adsorbente se obtuvo un espectro IR usando un Espectrómetro Nicolete Magna-IR 550 en un rango de frecuencia de $400-4000 \mathrm{~cm}^{-1}$. El DD se determinó utilizando la Ecuación (1) de Domszy y Roberts (1985) con los datos de absorbancia del Espectro IR a una longitud de onda de 3420 y $1660 \mathrm{~cm}^{-1}$.

$D D=100-\left[\frac{A_{1654}}{A_{3450}} \times 75.19\right]$

\section{b) Diseño experimental}

En la Tabla 1 se presentan los parámetros que se evaluaron para la cinética de adsorción de los iones metálicos con quitosano a partir de soluciones binarias.

Tabla 1 Parámetros considerados en la cinética de adsorción de $\mathrm{Cu}$ (II) y Hg (II).

\begin{tabular}{cccc}
\hline Tamaño & \multicolumn{2}{c}{$\begin{array}{c}\text { Concentración } \\
\text { de }\end{array}$} & $\begin{array}{c}\text { Velocidad } \\
\text { partícula }\end{array}$ \\
\cline { 2 - 3 } $\begin{array}{c}\text { de }) \\
(\mathrm{mm})\end{array}$ & $\mathrm{Cu}(\mathrm{II})$ & $\mathrm{Hg}(\mathrm{II})$ & $\begin{array}{c}\text { agitación } \\
(\text { rpm })\end{array}$ \\
\hline$<0.22$ & 1 & 1 & 300 \\
$0.22-0.45$ & 20 & 5 & 600 \\
& 50 & 10 & \\
\hline
\end{tabular}

Para el diseño experimental se usó el programa Statgraphics Plus for Windows 4.1. La clase de diseño fue Multi-factor categórico, el cual considera lo siguiente:

- Número de factores experimentales: 3

- Número de respuestas: 2

- Número de corridas: 24

- Grados de libertad para el error: 14

La Tabla 2 muestra los factores independientes a evaluar para el estudio de la adsorción. Mientras que la Tabla 3 muestra las variables dependientes (respuestas).

Tabla 2. Factores en el diseño experimental.

\begin{tabular}{ccc}
\hline Factores & Niveles & Unidades \\
\hline Concentración & 3 & $\mathrm{mg} / \mathrm{L}$ \\
Velocidad & 2 & $\mathrm{rpm}$ \\
Tamaño de & 2 & $\mathrm{~mm}$ \\
partícula & 2 & \\
\hline
\end{tabular}

Este es un diseño factorial estándar que consiste en todas las combinaciones de los niveles de los 
factores o variables independientes con un total de 24 corridas.

Tabla 3. Respuestas y sus unidades.

\begin{tabular}{cc}
\hline Respuestas & Unidades \\
\hline Comp_1 $(\mathrm{Cu})$ & $\mathrm{mg} / \mathrm{L}$ \\
Comp_2 $(\mathrm{Hg})$ & $\mathrm{mg} / \mathrm{L}$ \\
\hline
\end{tabular}

c) Experimentos de cinética de adsorción

Se prepararon $500 \mathrm{~mL}$ de soluciones de $\mathrm{Cu}$ (II) y $\mathrm{Hg}$ (II) y se vertieron en un vaso de precipitado de 2 L. El pH de la solución se ajustó a 6, añadiendo gotas de $\mathrm{NaOH} 0.3 \%$ y HCl $0.01 \mathrm{~N}$ ó $0.1 \mathrm{~N}$. Se adicionaron $2 \mathrm{~g}$ del adsorbente y la mezcla se agitó por 8 horas utilizando un agitador magnético (ver Fig. 2).

Durante el experimento se controló el pH de la solución para asegurar que no hubiera una disminución del $\mathrm{pH}$ durante el proceso. $\mathrm{El} \mathrm{pH}$ de la solución fue medido con un pH-metro Orión 410A.

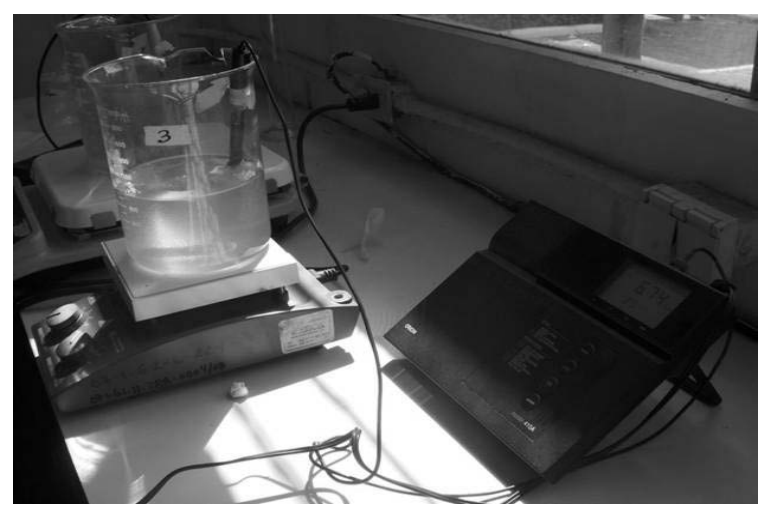

Fig. 2 Sistema utilizado para el estudio de la cinética de adsorción de los iones metálicos.

Cada período de tiempo (entre 2-120 min) se tomó una alícuota de $10 \mathrm{~mL}$, la cual fue filtrada para separar las trazas de adsorbente.

Las muestras fueron analizadas para determinar la concentración de iones $\mathrm{Cu}$ (II) usando un Espectrómetro de absorción atómica GBC 932 Plus con llama y de iones $\mathrm{Hg}$ (II) por la técnica de vapor frío usando un Generador de Hidruros HG3000.

\section{Modelos cinéticos}

Para identificar el mecanismo controlante en la velocidad de adsorción de $\mathrm{Cu}$ (II) y $\mathrm{Hg}$ (II) en el quitosano, se consideraron las siguientes etapas:

- La transferencia de masa del ión metálico del seno de la solución hacia la superficie del adsorbente.

- La adsorción del ión metálico en el adsorbente.

- La difusión interna del ión metálico en el adsorbente.

Para este propósito, los modelos simplificados de pseudo-primer orden y pseudo-segundo orden fueron aplicados para evaluar los datos experimentales obtenidos para cada par metálico (Gerente et al., 2007).

El conjunto de parámetros para los modelos de pseudo-primer orden (Ecuación 2) y pseudosegundo orden (Ecuación 3) fueron determinados por optimización no lineal, aplicando la función fminsearch, en el Programa MatLab®. Para evaluar cual de los modelos aplicados se ajustaba mejor a los datos experimentales, se hizo uso del coeficiente de Pearson $(R)$, el cual determina la correlación entre los datos experimentales y los datos calculados con los parámetros de cada modelo (Ecuación 4).

$$
\begin{aligned}
& \log \left(q_{e}-q_{t}\right)=\log \left(q_{e}\right)-\frac{k_{1}}{2.303} t \\
& \frac{t}{q_{t}}=\frac{1}{k_{2} q_{e}^{2}}+\frac{1}{q_{e}} t \\
& R=\frac{\sum(x-\bar{x})(y-\bar{y})}{\sqrt{\sum(x-\bar{x})^{2} \sum(y-\bar{y})^{2}}}
\end{aligned}
$$

\section{RESULTADOS Y DISCUSIÓN}

\section{Obtención del quitosano}

Los resultados mostraron que la producción de quitosano, por desacetilación básica de la quitina tuvo un porcentaje de rendimiento del $46.7 \%$. El DD del adsorbente es de $60 \%$. Este fue 
calculado por la Ecuación (1) con los datos de absorbancia del espectro IR de la Fig. 3 a una longitud de onda 3420 y $1660 \mathrm{~cm}^{-1}$.



Fig. 3 Espectro IR del quitosano obtenido a nivel de laboratorio.

Adsorción de los iones metálicos Cu(II) y Hg(II) de una mezcla binaria

La adsorción de cobre y mercurio en el quitosano se produce por el enlace entre el ión metálico con el grupo amino presente en el adsorbente. La adsorción puede ser por intercambio iónico, quelación o por atracción electrostática. El mecanismo de adsorción depende del $\mathrm{pH}$ y de la especiación de los iones metálicos en la solución (Vold et al., 2003).

El quitosano es una base débil y el equilibrio de disociación descrito por la Ecuación (5) depende del pH de la solución. A pH 6, aproximadamente el $50 \%$ de los grupos aminos se encuentran protonados en la forma de $\mathrm{NH}_{3}{ }^{+}$(Guibal, 2004).

$\mathrm{R}-\mathrm{NH}_{3}{ }^{+}+\mathrm{H}_{2} \mathrm{O} \leftrightarrows \mathrm{R}-\mathrm{NH}_{2}+\mathrm{H}_{3} \mathrm{O}^{+}$

La especiación de los iones $\mathrm{Cu}$ y $\mathrm{Hg}$ también depende del pH de la solución. Los resultados de la simulación de la especiación de estos iones con el programa PHREEQC a $\mathrm{pH} 6$ indica que las principales especies de $\mathrm{Hg}$ son: $\mathrm{HgCl}_{2}$, $\mathrm{Hg}(\mathrm{OH})_{2}$ y $\mathrm{HgClOH}$; mientras el cobre se encuentra como ion $\mathrm{Cu}^{2+}$ en la solución.

Esto sugiere que el principal mecanismo de adsorción para el $\mathrm{Hg}$ puede ser por atracción electrostática y para el $\mathrm{Cu}$, por quelación (Vieira et al., 2007).
Rhazi et al. (2002) propuso que el complejo $\mathrm{Cu}$ quitosano formado por el mecanismo de quelación depende del $\mathrm{pH}$ de la solución. Para $\mathrm{pH}>5.8$, el complejo $\left(\left[\mathrm{Cu}\left(-\mathrm{NH}_{2}\right)_{2}\right]^{2+}, 2 \mathrm{OH}\right)$ es considerado.

Los resultados de la adsorción de los iones $\mathrm{Cu}$ (II) y $\mathrm{Hg}$ (II) con quitosano a diferentes condiciones de trabajo mostraron que en la mayoría de los casos, ambos iones fueron adsorbidos por el quitosano, obteniéndose un porcentaje de adsorción entre $44-100 \%$ para $\mathrm{Cu}$ y entre $95-100 \%$ para el $\mathrm{Hg}$ (ver Tablas 4 y 5); obteniéndose los más altos porcentajes para el mercurio. Estos resultados también revelan que bajo estas condiciones, la presencia de un segundo ión metálico y la variación en las concentraciones iniciales de estos iones y el tamaño de partículas ejercen mayor influencia en la adsorción de $\mathrm{Cu}$ (II) que para la adsorción de $\mathrm{Hg}(\mathrm{II})$. Estos resultados son consistentes con los obtenidos por Vieira et al. (2007). Estos autores encontraron que los iones $\mathrm{Hg}$ fueron más eficazmente adsorbidos que los iones $\mathrm{Cu}$, indicando una fuerte interacción entre los iones $\mathrm{Hg}$ y el adsorbente.

Otros estudios han encontrado que el ion $\mathrm{Cu}^{2+}$ es preferentemente adsorbido en el quitosano en la presencia de los iones $\mathrm{Ni}^{2+}, \mathrm{Zn}^{2+}$ y $\mathrm{Cd}^{2+}$, y que esta selectividad incrementa cuando incrementa la fracción de cobre en la solución (Vold et al., 2003). Incluso esta preferencia por el ion $\mathrm{Cu}$ (II) es extendida cuando el ion esta simultáneamente presente con el $\mathrm{Co}$ (II) en quitosano modificado (Swayampakula et al., 2009). Sin embargo, en presencia del $\mathrm{Hg}$ la selectividad del quitosano por el $\mathrm{Cu}$ se ve significativamente afectada.

Las Figs. 3 y 4 muestran la velocidad de adsorción de $\mathrm{Cu}$ y $\mathrm{Hg}$, respectivamente, a partir de soluciones binarias (SB) y soluciones conteniendo un solo ión metálico (SS) a diferentes concentraciones iniciales y tamaño de partícula. Al comparar las velocidades de adsorción de ambos iones metálicos (Figs. 3 y 4), se observa que la velocidad de adsorción de los iones $\mathrm{Hg}$ (II) es más rápida y que la adsorción de los iones $\mathrm{Hg}$ alcanza más rápidamente el equilibrio. Esto puede deberse a la influencia que ejerce la concentración de 
ambos iones metálicos en la solución, ya que las concentraciones iniciales de $\mathrm{Hg}$ son más bajas permitiéndole adsorberse primero. Además, de la mayor preferencia o selectividad del quitosano por este ión y por la fuerte interacción entre este ión y el adsorbente (Vieira et al., 2007; Benavente, 2008).

Los gráficos de las Figs. 3 y 4 también muestran que para ambos iones la velocidad de adsorción es mayor cuando se tiene un solo ión en la solución (SS) que cuando se encuentran combinados (SB). No obstante, en el caso del $\mathrm{Hg}$ (II) al menor tamaño de partículas e independientemente de la concentración inicial del ion, la diferencia entre la velocidad de adsorción es menos pronunciada. Estos resultados revelan que la velocidad de adsorción de un ión metálico en el adsorbente es influenciada por la presencia de un segundo ión metálico en la solución debido a la competencia de ambos iones por los sitios de adsorción y a la selectividad del adsorbente por un ion con respecto a otro.

Efectos de los parámetros en la adsorción de $\mathrm{Cu}(\mathrm{II})$

La Tabla 4 presenta el porcentaje de adsorción de $\mathrm{Cu}(\mathrm{II})$ a diferentes concentraciones iniciales, tamaños de partículas del adsorbente y velocidad de agitación a partir de una solución binaria de iones $\mathrm{Cu}(\mathrm{II}) / \mathrm{Hg}$ (II). Estos resultados muestran que el porcentaje de adsorción del $\mathrm{Cu}$ se ve mayormente influenciado por la concentración inicial del ión metálico en la solución, ya que a medida que aumenta la concentración inicial de $\mathrm{Cu}$ (II) el porcentaje de adsorción disminuye.

A una concentración de $1 \mathrm{mg} / \mathrm{L}$ se obtuvieron altos porcentajes de adsorción (> 94\%) indicando que parámetros tales como la velocidad de agitación y el tamaño de partículas del adsorbente ejercen poca influencia en el porcentaje de adsorción de $\mathrm{Cu}$ (II). Así también, la presencia de otro ión en la solución no afectó significativamente la adsorción de $\mathrm{Cu}$ (II) debido principalmente a que había suficiente sitios activos disponibles en el adsorbente para la adsorción de ambos iones metálicos.
A $20 \mathrm{mg} / \mathrm{L}$ se alcanzaron porcentajes de adsorción mayores del $70 \%$, siendo el porcentaje más alto $(94.3 \%)$ cuando se trabajó con un tamaño de partículas de 0.22-0.45 mm y velocidad de agitación de $600 \mathrm{rpm}$. Estos resultados se contraponen con los encontrados por Benavente (2008) bajo las mismas condiciones de trabajo a partir de una solución conteniendo un solo ión. En este caso, el mayor porcentaje de adsorción (99.3\%) correspondió a $<0.22 \mathrm{~mm}$ y $600 \mathrm{rpm}$. Mientras a $50 \mathrm{mg} / \mathrm{L}$ se obtuvo un mayor porcentaje de adsorción cuando se trabajó con un tamaño de partículas < $0.22 \mathrm{~mm}$ y velocidad de agitación de $300 \mathrm{rpm}$. Es decir, el porcentaje de adsorción se ve más afectado por la velocidad de agitación y el tamaño de partículas a medida que incrementa la concentración inicial de $\mathrm{Cu}(\mathrm{II})$.

\section{Cinética de adsorción de Cu(II)}

La Fig. 5 muestra la capacidad de adsorción (qt) de $\mathrm{Cu}(\mathrm{II})$ en función del tiempo a diferentes concentraciones iniciales, tamaños de partículas del adsorbente y velocidad de agitación a partir de una solución binaria de iones $\mathrm{Cu}(\mathrm{II}) / \mathrm{Hg}$ (II). Los gráficos muestran que a medida que la concentración de $\mathrm{Cu}$ (II) aumenta en la solución, la velocidad de agitación y el tamaño de partículas tienen mayor influencia en la velocidad de adsorción. A una concentración inicial de $1 \mathrm{mg} / \mathrm{L}$ (Fig. 5(a)), la velocidad de adsorción es más rápida y los parámetros de velocidad de agitación y tamaño de partículas, así como también la presencia de un segundo ión metálico, no tienen mucha influencia en la velocidad de adsorción.

En la Fig. 5(b) se puede observar que para una concentración inicial de $20 \mathrm{mg} / \mathrm{L}$ la velocidad de adsorción es más lenta que para $1 \mathrm{mg} / \mathrm{L}$. A esta concentración la velocidad de agitación y el tamaño de partículas influyen grandemente en la velocidad de adsorción dando como resultado, que al tamaño de partículas entre $0.22-0.45 \mathrm{~mm}$ y a una velocidad de agitación de $300 \mathrm{rpm}$ la velocidad de adsorción disminuye notablemente, observándose que bajo estas condiciones no se había alcanzado el equilibrio en un tiempo de 480 minutos. 
I. Matus et al.

Tabla 4 Porcentaje de adsorción y parámetros de los modelos cinéticos del $\mathrm{Cu}(\mathrm{II})$.

\begin{tabular}{|c|c|c|c|c|c|c|c|c|c|}
\hline \multirow{2}{*}{$\begin{array}{c}\text { Concentración } \\
\text { inicial } \\
(m g / L)\end{array}$} & \multirow{2}{*}{$\begin{array}{l}\text { Tamaño de } \\
\text { partícula } \\
\text { (mm) }\end{array}$} & \multirow{2}{*}{$\begin{array}{l}\text { Velocidad de } \\
\text { agitación } \\
\text { (rpm) }\end{array}$} & \multirow{2}{*}{$\begin{array}{l}\text { Porcentaje } \\
\text { de } \\
\text { adsorción }\end{array}$} & \multicolumn{3}{|c|}{ Modelo de pseudo-primer orden } & \multicolumn{3}{|c|}{ Modelo de pseudo-segundo orden } \\
\hline & & & & $R$ & $\mathrm{q}_{\mathrm{e}}$ & $\mathrm{k}_{1}$ & $R$ & $\mathrm{q}_{\mathrm{e}}$ & $\mathrm{k}_{2}$ \\
\hline 1.0 & $<0.22$ & 300 & 100.00 & 0.983 & 0.480 & 0.530 & 0.996 & 0.494 & 1.965 \\
\hline 1.0 & $<0.22$ & 600 & 94.56 & 0.996 & 0.409 & 1.072 & 0.998 & 0.414 & 8.259 \\
\hline 1.0 & $0.22-0.45$ & 300 & 98.58 & 0.981 & 0.437 & 0.371 & 0.996 & 0.452 & 1.478 \\
\hline 1.0 & $0.22-0.45$ & 600 & 98.52 & 0.982 & 0.402 & 0.327 & 0.996 & 0.417 & 1.394 \\
\hline 20.0 & $<0.22$ & 300 & 93.69 & 0.962 & 8.958 & 0.032 & 0.983 & 9.936 & $4.30 \times 10^{-3}$ \\
\hline 20.0 & $<0.22$ & 600 & 91.49 & 0.959 & 8.550 & 0.040 & 0.983 & 9.373 & $6.00 \times 10^{-3}$ \\
\hline 20.0 & $0.22-0.45$ & 300 & 70.17 & 0.952 & 5.671 & 0.018 & 0.970 & 6.538 & $3.30 \times 10^{-3}$ \\
\hline 20.0 & $0.22-0.45$ & 600 & 94.32 & 0.979 & 9.266 & 0.013 & 0.986 & 11.045 & $1.30 \times 10^{-3}$ \\
\hline 50.0 & $<0.22$ & 300 & 91.38 & 0.957 & 18.746 & 0.025 & 0.978 & 21.199 & $1.50 \times 10^{-3}$ \\
\hline 50.0 & $<0.22$ & 600 & 87.61 & 0.963 & 17.538 & 0.024 & 0.983 & 19.876 & $1.50 \times 10^{-3}$ \\
\hline 50.0 & $0.22-0.45$ & 300 & 43.95 & 0.978 & 8.628 & 0.010 & 0.983 & 10.568 & $9.72 \times 10^{-4}$ \\
\hline 50.0 & $0.22-0.45$ & 600 & 55.18 & 0.994 & 13.884 & 0.004 & 0.994 & 19.701 & $1.64 \times 10^{-4}$ \\
\hline
\end{tabular}

Tabla 5 Porcentaje de adsorción y parámetros de los modelos cinéticos del $\mathrm{Hg}(\mathrm{II})$.

\begin{tabular}{|c|c|c|c|c|c|c|c|c|c|}
\hline \multirow{2}{*}{$\begin{array}{l}\text { Concentración } \\
\text { inicial } \\
(\mathrm{mg} / \mathrm{L})\end{array}$} & \multirow{2}{*}{$\begin{array}{l}\text { Tamaño de } \\
\text { partícula } \\
(\mathrm{mm})\end{array}$} & \multirow{2}{*}{$\begin{array}{l}\text { Velocidad de } \\
\text { agitación } \\
\text { (rpm) }\end{array}$} & \multirow{2}{*}{$\begin{array}{c}\text { Porcentaje } \\
\text { de } \\
\text { adsorción }\end{array}$} & \multicolumn{3}{|c|}{ Modelo de pseudo-primer orden } & \multicolumn{3}{|c|}{ Modelo de pseudo-segundo orden } \\
\hline & & & & $R$ & $q_{e}$ & $\mathrm{k}_{1}$ & $R$ & $\mathrm{q}_{\mathrm{e}}$ & $\mathrm{k}_{2}$ \\
\hline 1.0 & $<0.22$ & 300 & 100.00 & 1.000 & 0.482 & 2.115 & 1.000 & 0.483 & 59.372 \\
\hline 1.0 & $<0.22$ & 600 & 99.84 & 1.000 & 0.464 & 2.579 & 1.000 & 0.464 & 151.723 \\
\hline 1.0 & $0.22-0.45$ & 300 & 99.85 & 1.000 & 0.528 & 2.090 & 1.000 & 0.529 & 56.549 \\
\hline 1.0 & $0.22-0.45$ & 600 & 99.71 & 1.000 & 0.532 & 18.984 & 1.000 & 0.532 & 110.160 \\
\hline 5.0 & $<0.22$ & 300 & 99.98 & 0.963 & 3.878 & 0.368 & 0.984 & 4.007 & 0.176 \\
\hline 5.0 & $<0.22$ & 600 & 99.97 & 0.996 & 5.252 & 0.749 & 1.000 & 5.351 & 0.327 \\
\hline 5.0 & $0.22-0.45$ & 300 & 98.84 & 0.977 & 1.738 & 0.142 & 0.996 & 1.833 & 0.134 \\
\hline 5.0 & $0.22-0.45$ & 600 & 98.82 & 0.970 & 2.017 & 0.199 & 0.995 & 2.130 & 0.149 \\
\hline 20.0 & $<0.22$ & 300 & 99.58 & 0.989 & 9.577 & 0.340 & 1.000 & 9.940 & 0.061 \\
\hline 10.0 & $<0.22$ & 600 & 99.63 & 0.979 & 5.418 & 0.290 & 0.993 & 5.628 & 0.094 \\
\hline 10.0 & $0.22-0.45$ & 300 & 94.96 & 0.889 & 4.227 & 0.105 & 0.904 & 4.630 & 0.028 \\
\hline 10.0 & $0.22-0.45$ & 600 & 97.58 & 0.988 & 4.774 & 0.024 & 0.987 & 5.438 & 0.005 \\
\hline
\end{tabular}

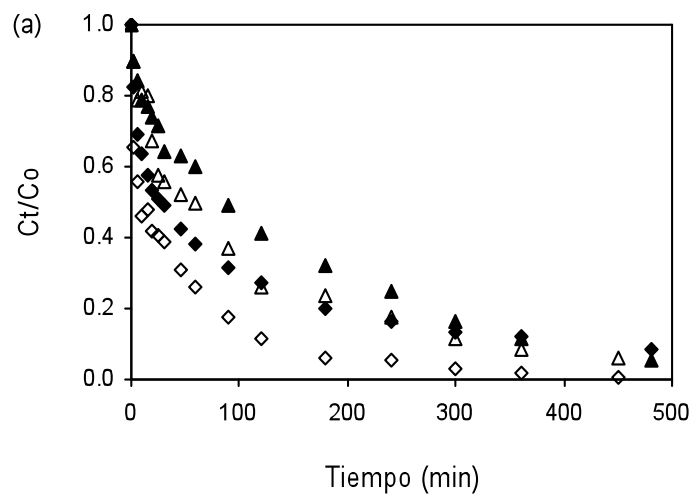

$\bullet<0.22 \mathrm{~mm}$ (S.B) $\quad \Delta 0.22-0.45 \mathrm{~mm}(\mathrm{~S} . \mathrm{B})$

$\diamond<0.22 \mathrm{~mm}$ (S.S) $\quad \Delta 0.22-0.45 \mathrm{~mm}$ (S.S)

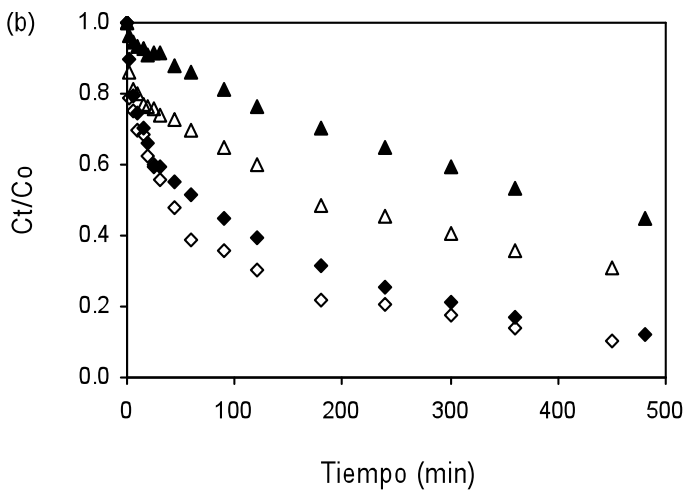

$\bullet<0.22 \mathrm{~mm}(\mathrm{~S} . \mathrm{B}) \quad \Delta 0.22-0.45 \mathrm{~mm}(\mathrm{~S} . \mathrm{B})$

$\diamond<0.22 \mathrm{~mm}$ (S.S) $\quad \Delta 0.22-0.45 \mathrm{~mm}$ (S.S)

Fig. 3 Comparación de la velocidad de adsorción de Cu (II) a partir de soluciones binarias (S.B) y soluciones con un solo ión (S.S), a $600 \mathrm{rpm}$ y a diferentes concentraciones iniciales: (a) $20 \mathrm{mg} / \mathrm{L}$ y (b) $50 \mathrm{mg} / \mathrm{L}$. 
(a)

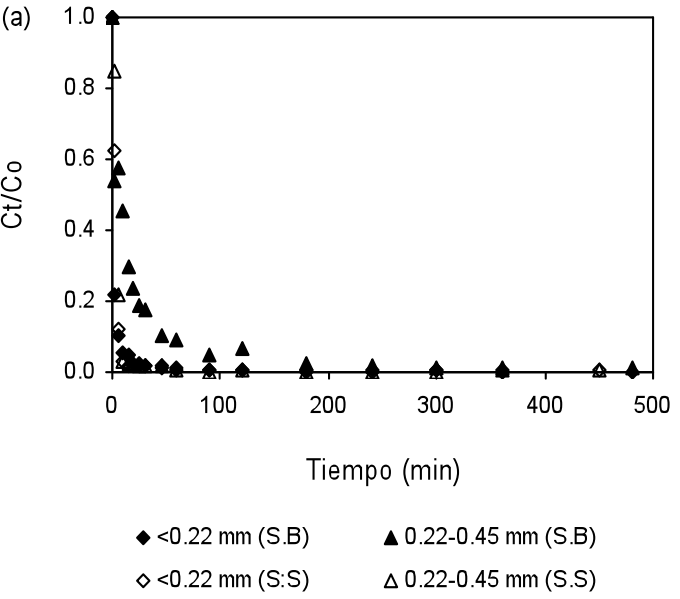

(b)

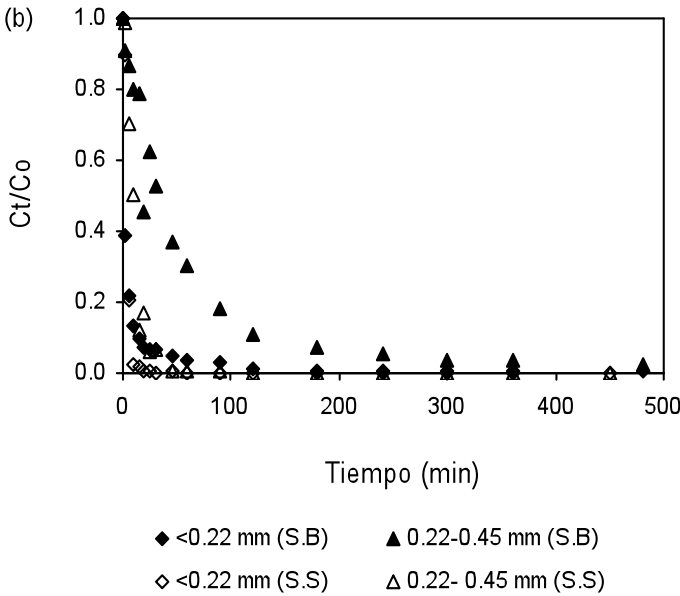

Fig. 4 Comparación de la velocidad de adsorción de $\mathrm{Hg}$ (II) a partir de soluciones binarias (S.B) y soluciones con un solo ión (S.S), a $600 \mathrm{rpm}$ y a diferentes concentraciones iniciales: (a) $5 \mathrm{mg} / \mathrm{L}$ y (b) $20 \mathrm{mg} / \mathrm{L}$.

Por otro lado, a concentraciones de $50 \mathrm{mg} / \mathrm{L}$ la velocidad de adsorción es aún más lenta, ya que aún después de transcurridos los 480 minutos todavía la reacción no había alcanzado el equilibrio. En la Fig. 5(c) se observa que al menor tamaño de partículas se obtuvo una velocidad de adsorción más rápida que cuando se trabajó con el mayor tamaño de partículas, independientemente de la velocidad de agitación, ya que la influencia de este parámetro no es tan evidente como el que ejerce el tamaño de partículas.

\section{Modelos cinéticos para la adsorción del Cu(II)}

La evaluación de los modelos cinéticos a los datos experimentales se realizó para determinar cuál de los modelos propuestos es el que mejor describe la cinética de adsorción y el paso limitante en la adsorción del ión metálico a partir de una solución binaria. La Tabla 4 muestra los parámetros de los modelos de pseudo-primer orden y pseudo-segundo orden, así como el coeficiente de correlación de Pearson $(R)$ para el ión $\mathrm{Cu}$ a diferentes concentraciones iniciales, tamaño de partícula y velocidad de agitación.

Al evaluar los datos experimentales con una concentración inicial de $1 \mathrm{mg} / \mathrm{L}, 20 \mathrm{mg} / \mathrm{L}$ y 50 $\mathrm{mg} / \mathrm{L}$, independientemente del tamaño de partícula y la velocidad de agitación, se obtuvo que el modelo cinético que mejor describe la cinética de adsorción es el modelo de pseudosegundo orden; por lo tanto, el mecanismo controlante de la velocidad de adsorción es la reacción de adsorción del ion $\mathrm{Cu}$ (II) en el adsorbente y no la transferencia de masa. Los parámetros de $\mathrm{q}_{\mathrm{e}} \mathrm{y} \mathrm{k}_{1}$ para el modelo de pseudoprimer orden y los parámetros de $\mathrm{q}_{\mathrm{e}} \mathrm{y} \mathrm{k}_{2}$ para el modelo de pseudo-segundo orden se muestran en la Tabla 4, los cuales fueron usados para comprobar el ajuste de cada modelo a los datos experimentales. Los resultados muestran que el valor de $\mathrm{k}_{2}$ disminuye a medida que incrementa la concentración del ión metálico.

La Fig. 5 presenta los gráficos de la capacidad de adsorción (qt) en función del tiempo a diferentes concentraciones iniciales de 1,20 y $50 \mathrm{mg} / \mathrm{L}$. Se puede observar el ajuste de los modelos de pseudo-primer orden y pseudosegundo orden a los datos experimentales. Los gráficos muestran que hay un buen ajuste de los resultados experimentales al modelo de pseudosegundo orden. Estos resultados se contraponen con los encontrados por Swayampakula et al. (2009) ya que ellos determinaron que en soluciones binarias $\mathrm{Cu}-\mathrm{Ni}$ y $\mathrm{Cu}-\mathrm{Co}$, el proceso de adsorción de los iones $\mathrm{Cu}$ (II) en quitosano modificado sigue la cinética de primer orden. Esto significa que el paso controlante en la velocidad de adsorción dependerá de varios factores: la presencia del otro ion en la solución binaria, la selectividad del quitosano por el ion y el tipo y características del adsorbente. 
I. Matus et al.
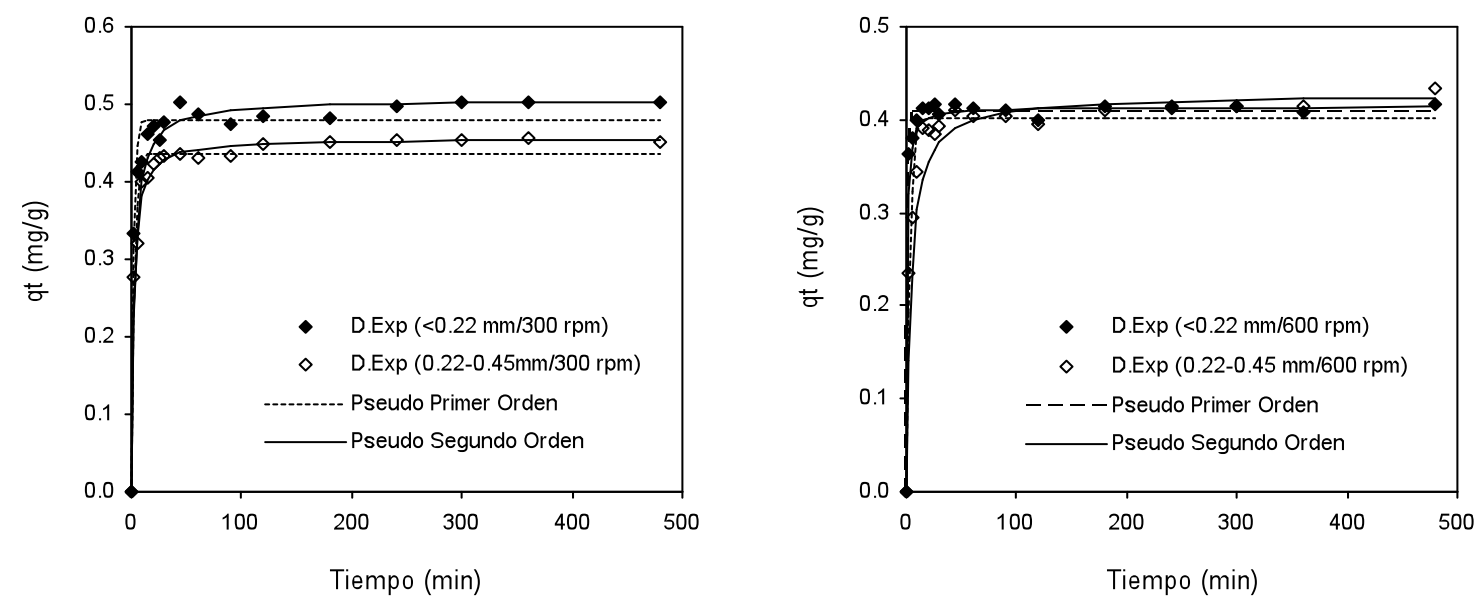

(a)
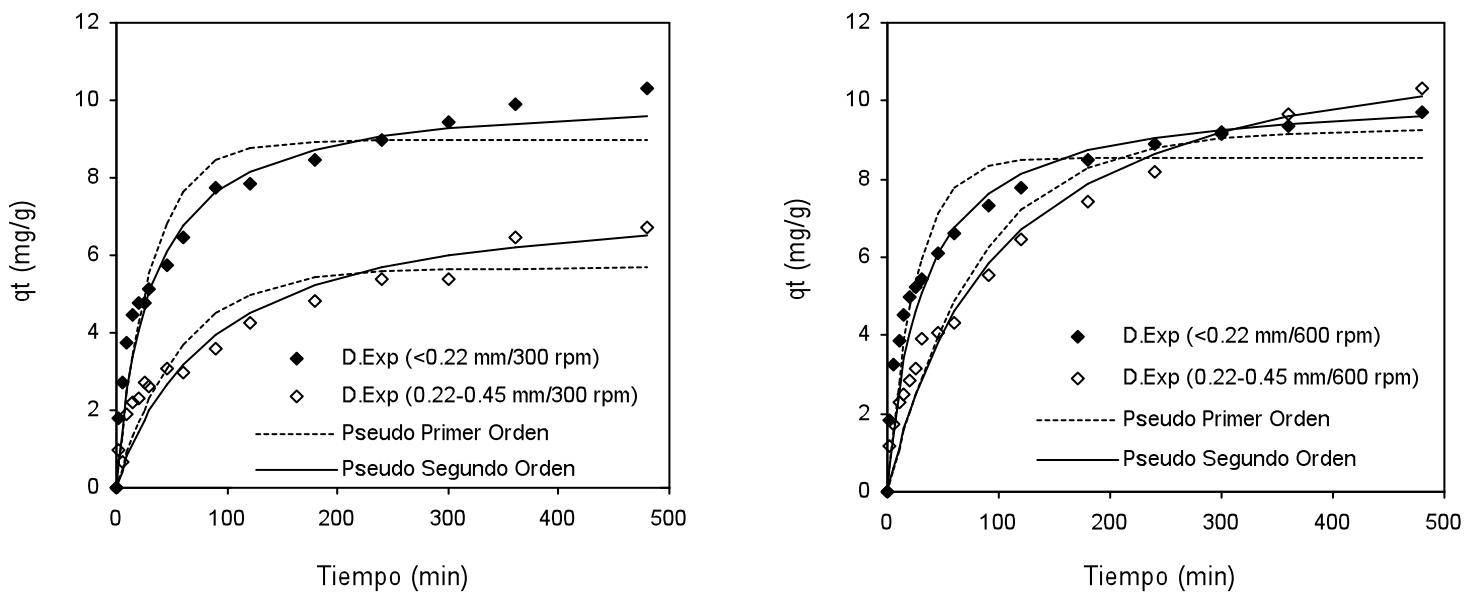

(b)
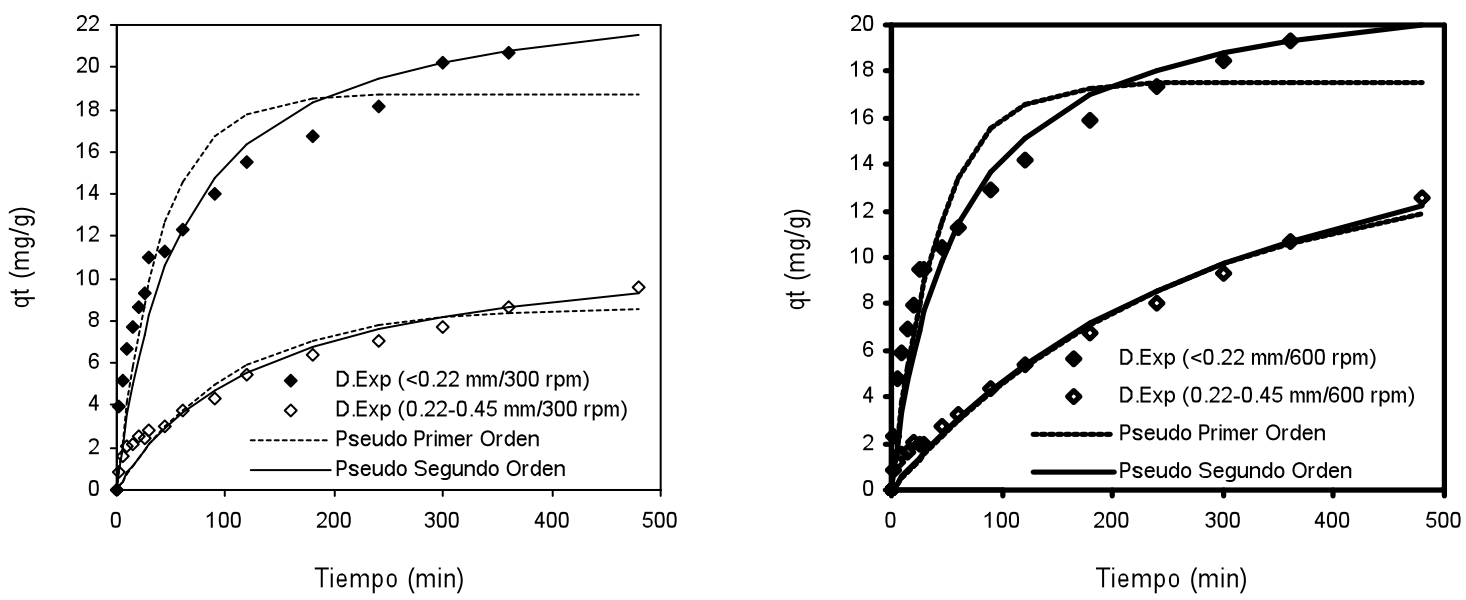

(c)

Fig. 5 Datos experimentales vs. modelo de pseudo-primer orden y pseudo-segundo orden de $\mathrm{Cu}(\mathrm{II})$ a (a) $\mathrm{C}_{\mathrm{o}}=1 \mathrm{mg} / \mathrm{L}$, (b) $\mathrm{C}_{\mathrm{o}}=20 \mathrm{mg} / \mathrm{L}$ y (c) $\mathrm{C}_{\mathrm{o}}=50 \mathrm{mg} / \mathrm{L}$ a diferentes tamaños de partícula y velocidad de agitación de 300 rpm (izquierda) y 600 rpm (derecha). 
Efectos de los parámetros en la adsorción de $\mathrm{Hg}(\mathrm{II})$

La adsorción de $\mathrm{Hg}$ (II) se ve afectada por la concentración inicial, ya que a medida que la concentración aumenta el porcentaje de adsorción disminuye. El tamaño de partículas y la velocidad de agitación también juegan un papel fundamental, porque al menor tamaño de partículas $(<0.22 \mathrm{~mm})$ y mayor velocidad de agitación $(600 \mathrm{rpm})$ se produce un mayor porcentaje de adsorción (ver Tabla 5).

En soluciones con concentración inicial de 1 $\mathrm{mg} / \mathrm{L}$ de $\mathrm{Hg}$ (II), se obtuvo un alto porcentaje de adsorción (aproximadamente el $100 \%$ ). Este resultado fue independiente de la velocidad de agitación y del tamaño de partículas; es decir, que a baja concentraciones los parámetros antes señalados tienen poca influencia en la adsorción. Esto puede deberse a la alta afinidad que tiene el quitosano por este ión metálico.

Por otro lado, la Fig. 6(a) muestra que el proceso de adsorción se da de manera rápida, alcanzándose de forma casi instantánea el equilibrio, independientemente del tamaño de partículas y la velocidad de agitación.

A concentraciones iniciales de $5 \mathrm{mg} / \mathrm{L}$ también se alcanza un alto porcentaje de adsorción (aproximadamente el $99 \%$ ). Sin embargo, se observa que al mayor tamaño de partículas (0.22-0.45 mm), a las dos diferentes velocidades de agitación, la adsorción es ligeramente más baja; lo cual indica que el tamaño de partículas influye en el proceso de adsorción. Por otro lado, a $20 \mathrm{mg} / \mathrm{L}$ se logra un mayor porcentaje adsorción a menor tamaño de partículas y a las dos diferentes velocidades de agitación. También se puede observar que a mayor tamaño de partículas $(0.22-0.45 \mathrm{~mm})$ y a las diferentes velocidades de agitación el porcentaje de adsorción ligeramente disminuye. Esto indica que, al igual que en el caso anterior, el tamaño de partículas juega un papel predominante en el proceso de adsorción a medida que incrementa la concentración inicial del ión $\mathrm{Hg}$.

Las Fig. 6(b) y Fig. 6(c) correspondientes a 5 y $20 \mathrm{mg} / \mathrm{L}$, respectivamente, muestran que la velocidad de adsorción es notablemente rápida en los primeros 50 minutos y se vuelve gradualmente lenta hasta alcanzar el equilibrio. Se puede observar que el tamaño de partículas tiene gran influencia, ya que a menor tamaño de partículas la velocidad de adsorción ocurre de manera más rápida, independientemente de las velocidades de agitación. Esto puede deberse a la poca porosidad del quitosano, lo cual permite que se saturen los sitios activos externos y luego se produzca un lento decrecimiento de la velocidad de adsorción generado por el impedimento estérico de las especies hidrolizadas $\mathrm{H}_{3} \mathrm{O}^{+}$que son liberadas en el intercambio iónico (Flores et al., 2005).

\section{Modelos cinéticos para la adsorción de $\mathrm{Hg}(\mathrm{II})$}

En la aplicación de los modelos cinéticos para la concentración inicial de $1 \mathrm{mg} / \mathrm{L}$ se obtuvo, de manera general, que los datos se ajustaron a ambos modelos, dando un coeficiente de correlación de aproximadamente uno (ver Tabla 6). Ya que el coeficiente de correlación $r$ no fue suficiente para evaluar cuantitativamente la validez de la predicción del modelo, se usó otra herramienta estadística para esta evaluación: el porcentaje de desviación estándar $(\% \Delta \mathrm{q})$ entre los resultados calculados y experimentales (Juang y Shao, 2002):

$$
\Delta q(\%)=100 \times \sqrt{\frac{\sum\left[\left(q_{\text {expt }}-q_{\text {calc }}\right) / q_{\text {expt }}\right]^{2}}{(N-1)}}
$$

Los resultados obtenidos se reflejan en la Tabla 6. En esta tabla se puede observar que el porcentaje de desviación estándar para concentraciones iniciales de $1 \mathrm{mg} / \mathrm{L}$ es más bajo para el modelo de pseudo-primer orden en los primeros $30 \mathrm{~min}$. Esto indica que el modelo que mejor describe la cinética de adsorción en este rango de tiempo es el modelo de pseudo-primer orden, y que por lo tanto el mecanismo controlante en el proceso de adsorción es la transferencia de masa. Sin embargo, para rangos de tiempo mayores que $30 \mathrm{~min}$., se observa que el $\% \Delta \mathrm{q}$ es menor para el modelo de segundo orden, lo que indica que el mecanismo controlante es la adsorción química. 
I. Matus et al.
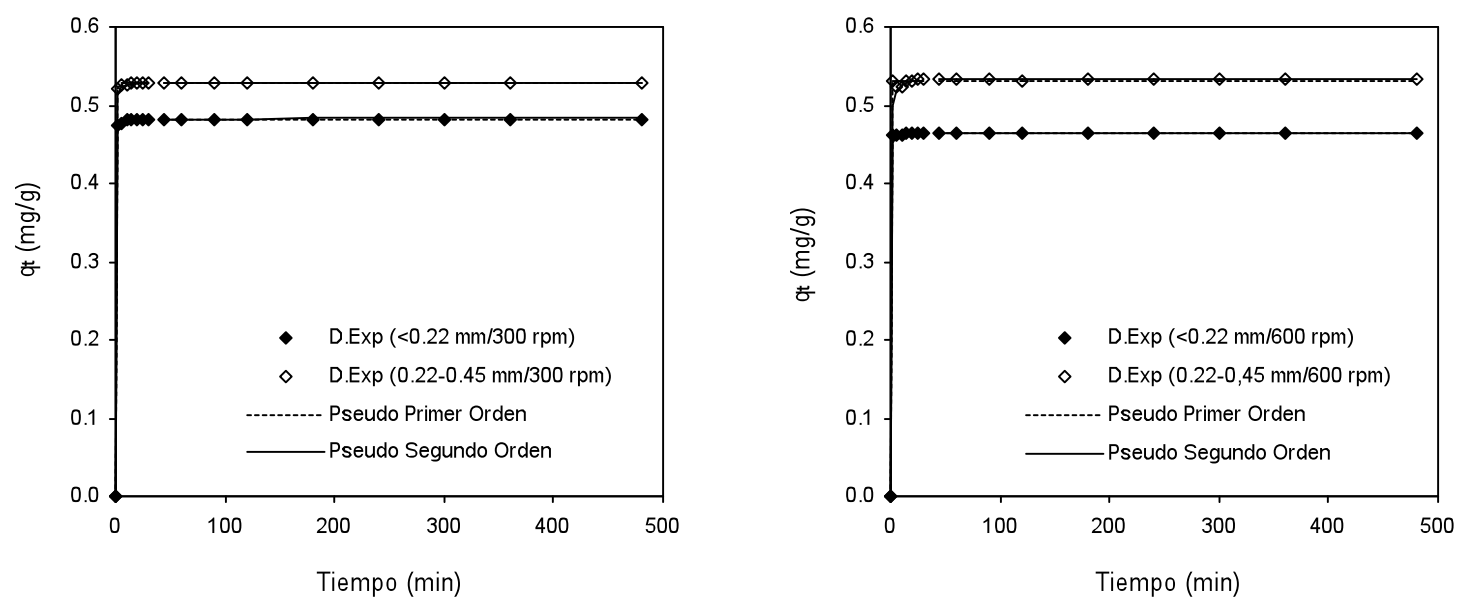

(a)
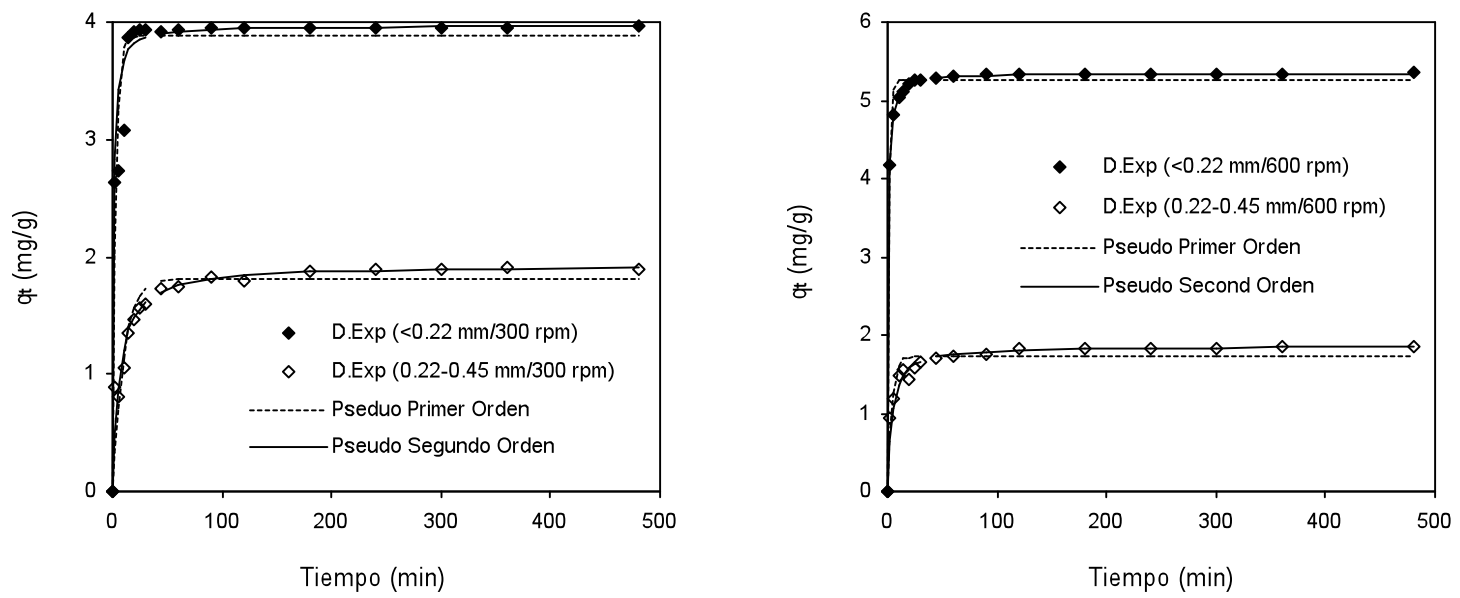

(b)
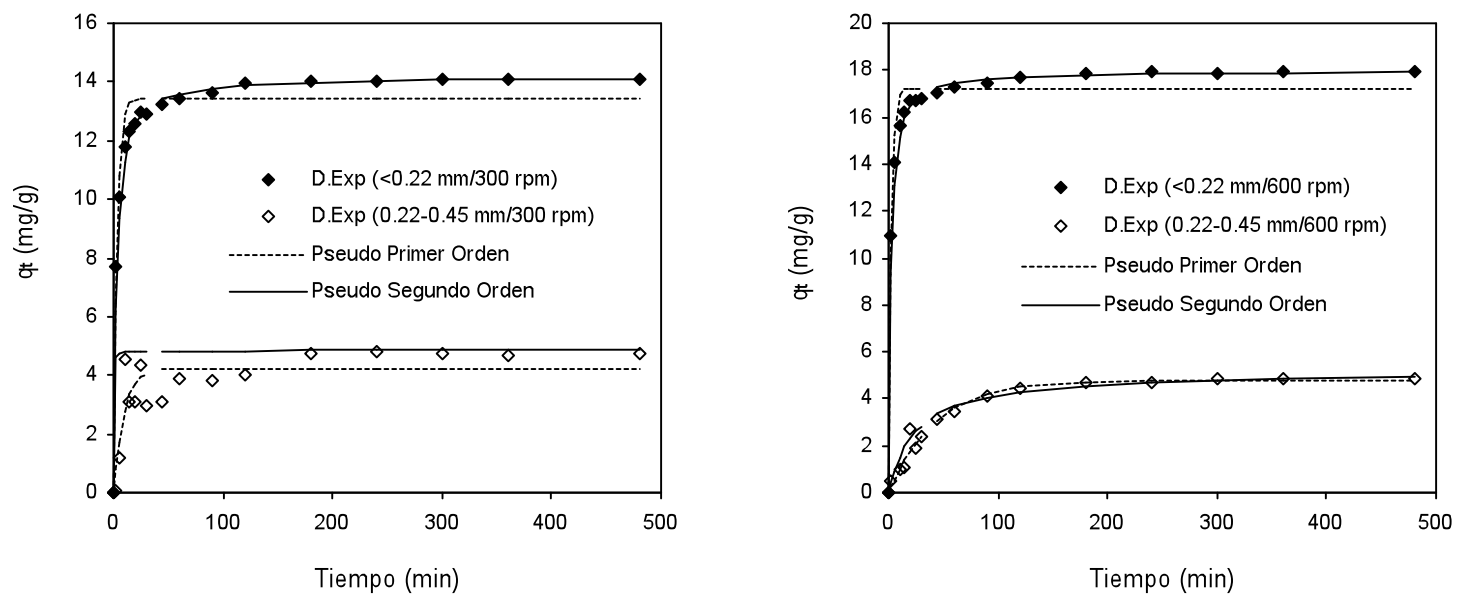

(c)

Fig. 6 Datos experimentales vs. Modelo de pseudo primer orden y pseudo segundo orden de $\mathrm{Hg}$ (II) a (a) $\mathrm{C}_{0}=1 \mathrm{mg} / \mathrm{L}$, (b) $\mathrm{C}_{\mathrm{o}}=5 \mathrm{mg} / \mathrm{L}$ y (c) $\mathrm{C}_{0}=20 \mathrm{mg} / \mathrm{L}$ a diferentes tamaños de partícula y velocidad de agitación de $300 \mathrm{rpm}$ (izquierda) y $600 \mathrm{rpm}$ (derecha). 
Tabla 6 Porcentaje de desviación estándar para $\mathrm{Co}=1 \mathrm{mg} / \mathrm{L} \mathrm{de} \mathrm{Hg}$ (II).

\begin{tabular}{ccccccc}
\hline & & \multicolumn{3}{c}{$\begin{array}{c}\text { Porcentaje de desviación } \\
\text { estándar }(\% \Delta q)\end{array}$} \\
\cline { 3 - 7 } $\begin{array}{c}\text { Tamaño de } \\
\text { partícula } \\
(\text { mm })\end{array}$ & $\begin{array}{c}\text { Velocidad } \\
\text { de agitación } \\
\text { (rpm) }\end{array}$ & \multicolumn{3}{c}{$\begin{array}{c}\text { Modelo pseudo } \\
\text { primer orden }\end{array}$} & $\begin{array}{c}\text { Modelo pseudo } \\
\text { segundo orden }\end{array}$ \\
\cline { 3 - 7 } & & $30 \mathrm{~min}$ & $>30 \mathrm{~min}$ & $30 \mathrm{~min}$ & $>30 \mathrm{~min}$ \\
\hline$<0.22$ & 300 & 0.248 & 0.083 & 0.290 & 0.038 \\
$<0.22$ & 600 & 0.113 & 0.080 & 0.552 & 0.074 \\
$0.22-0.45$ & 300 & 0.150 & 0.117 & 0.329 & 0.028 \\
$0.22-0.45$ & 600 & 0.582 & 0.209 & 1.675 & 0.088 \\
\hline
\end{tabular}

En la evaluación de los modelos cinéticos para concentraciones iniciales de $5 \mathrm{mg} / \mathrm{L}$ y $10 \mathrm{mg} / \mathrm{L}$, se observa que en la mayoría de los casos los valores de $\mathrm{r}$ son mayores para el modelo de pseudo-segundo orden, por lo tanto este modelo es el que mejor describe la cinética de adsorción para todos los parámetros evaluados. En estos casos, el paso controlante es la adsorción química y no la transferencia de masa. Para una concentración de $20 \mathrm{mg} / \mathrm{L}$ y tamaño de partículas entre $0.22-0.45 \mathrm{~mm}$, independientemente de la velocidad de agitación, el modelo que mejor describe la cinética de adsorción es el modelo de pseudoprimer orden.

En la Fig. 6 se presentan los gráficos de la capacidad de adsorción $\left(\mathrm{q}_{\mathrm{t}}\right)$ en función del tiempo a diferentes concentraciones iniciales, en los que se observa el ajuste de los modelos de pseudo-primer orden y pseudo-segundo orden a los datos experimentales.

\section{CONCLUSIONES}

El estudio mostró que el rendimiento de obtención del quitosano a nivel de laboratorio fue de $46.7 \%$, con un grado de desacetilación de $60 \%$.

Los parámetros evaluados tales como concentración inicial, tamaño de partículas y velocidad de agitación ejercen influencia en la capacidad de adsorción, ya que para $\mathrm{Cu}$ (II) y $\mathrm{Hg}(\mathrm{II})$, se obtienen mayores porcentajes de adsorción a tamaños de partículas $<0.22 \mathrm{~mm}$ y velocidad de agitación de 600 rpm. Además se comprobó que el grado de afinidad del quitosano con los iones metálicos, cuando se trabaja con soluciones binarias, sigue la secuencia: $\mathrm{Hg}>\mathrm{Cu}$.

La cinética de adsorción del $\mathrm{Cu}(\mathrm{II})$, en la mayoría de los casos, se ajusta mejor al modelo de pseudo-segundo orden, indicando que en la reacción global de adsorción, el paso controlante en la reacción global es la adsorción y no la transferencia de masa. Para el $\mathrm{Hg}$ (II) a concentraciones de $1 \mathrm{mg} / \mathrm{L}, \quad$ los datos experimentales se ajustaron a ambos modelos; mientras que a $5 \mathrm{mg} / \mathrm{L}$ y $20 \mathrm{mg} / \mathrm{L}$, el modelo que mejor describe la cinética de adsorción es el modelo de pseudo-segundo orden.

\section{AGRADECIMIENTOS}

Los autores agradecen a la Agencia Sueca de Cooperación Internacional para el Desarrollo, (Asdi) y a la Universidad Nacional de Ingeniería (UNI), por el apoyo brindado para la realización de este trabajo.

\section{NOTACIÓN}

\begin{tabular}{|c|c|c|}
\hline$A_{1654}$ & $\begin{array}{l}\text { Absorbancia a la longitud } \\
\text { de onda de } 1654 \mathrm{~nm}\end{array}$ & $(-)$ \\
\hline$A_{3450}$ & $\begin{array}{l}\text { Absorbancia a la longitud } \\
\text { de onda de } 3450 \mathrm{~nm}\end{array}$ & $(-)$ \\
\hline$D D$ & Grado de desacetilación & $(\%)$ \\
\hline$k_{1}$ & $\begin{array}{l}\text { Constante de la velocidad } \\
\text { de reacción de pseudo- } \\
\text { primer orden }\end{array}$ & $(1 / \mathrm{min})$ \\
\hline$k_{2}$ & $\begin{array}{l}\text { Constante de la velocidad } \\
\text { de reacción de pseudo- } \\
\text { segundo orden }\end{array}$ & $\frac{(\mathrm{g} /(\mathrm{mg}}{\min ))}$ \\
\hline$q_{e}$ & $\begin{array}{l}\text { Capacidad de adsorción } \\
\text { en el equilibrio }\end{array}$ & $(\mathrm{mg} / \mathrm{g})$ \\
\hline$q_{t}$ & $\begin{array}{l}\text { Capacidad de adsorción } \\
\text { en un tiempo t }\end{array}$ & $(\mathrm{mg} / \mathrm{g})$ \\
\hline$R$ & Coeficiente de Pearson & \\
\hline
\end{tabular}

\section{REFERENCIAS}

Álvarez, E. (2007). Determinación de Isotermas de Adsorción de Hierro, Cobre y Zinc en Quitosana. Tesis de Ingeniero Químico. Universidad Nacional de Ingeniería (UNI), Managua, Nicaragua. 
Benavente, M. (2008), Adsorption of Metallic Ions onto Chitosan: Equilibrium and Kinetic Studies. Licentiate Thesis. The Royal Institute of Technology (KTH), Stockholm, Sweden.

Benavente, M., Sjörén, A. \& Martínez, J. (2007). Remoción de mercurio de efluentes mineros por biosorción: un caso de estudio en la ciudad de La Libertad, Chontales, Nicaragua. Nexo, Vol. 20, No. 2, pp. 47-55.

Domszy, J.G. \& Roberts, G.A.F. (1985). Evaluation of Infrared Spectroscopic Techniques for Analysing Chitosan. Die Makromolekulare Chemie, Vol. 186, pp. 1671-1677. doi: $\underline{10.1002 / \mathrm{macp} .1985 .021860815}$

Flores, J.A., Navarro, A.E., Ramos, K.P., Chang, L., Ale, N., Ly, M., \& Maldonado, H.J. (2005). Adsorción de $\mathrm{Cu}$ (II) por quitosano en Polvo y Perlas de Gel. Revista de la Sociedad Química del Perú, Vol. 71, No. 1, pp. 17-25.

Gerente, C., Lee, V.K.C., Cloirec, P.L., \& McKay, G. (2007). Application of Chitosan for the Removal of Metals From Wastewaters by Adsorption-Mechanisms and Models Review. Critical Reviews in Environmental Science and Technology, Vol. 37, No. 1, pp. 41-127. doi: $\underline{10.1080 / 10643380600729089}$

Guibal, E. (2004). Interactions of metal ions with chitosan-based sorbents: a review. Separation and Purification Technology, Vol. 38, No. 1, pp. 43-74.

Hernández, D. \& Escorcia, D. (2009). Propuesta Técnica para la obtención de quitina a partir de caparazones de crustáceos a nivel de planta piloto. Tesis de Ingeniero Químico. Universidad Nacional de Ingeniería (UNI), Managua, Nicaragua.

Juang, R.-S., \& Shao, H.-J. (2002). Effect of pH on Competitive Adsorption of $\mathrm{Cu}(\mathrm{II}), \mathrm{Ni}$ (II), and $\mathrm{Zn}$ (II) from Water onto Chitosan Beads. Adsorption, Vol. 8, No. 1, pp. 71-78. doi: 10.1023/A:1015222607996

Muzzarelli, R.A.A. (1977). Chitin. Oxford, UK: Pergamon Press.
Pastor de Abram, A. (2004). Quitina y Quitosano: obtención, caracterización y aplicaciones. Lima, Peru: Fondo Editorial de la Pontifica Universidad Católica del Peru.

Rhazi, M., Desbrières, J., Tolaimate, A., Rinaudo, M., Vottero, P., \& Alagui, A. (2002). Contribution to the study of the complexation of copper by chitosan and oligomers. Polymer, Vol. 43, No. 4, pp. 1267-1276. doi: 10.1016/S0032$\underline{3861(01) 00685-1}$

Swayampakula, K., Boddu, V.M., Nadavala, S.K., \& Abburi, K. (2009). Competitive adsorption of $\mathrm{Cu}$ (II), Co (II) and Ni (II) from their binary and tertiary aqueous solutions using chitosan-coated perlite beads as biosorbent. Journal of Hazardous Materials, Vol. 170, No. 2-3, pp. 680-689.

Vieira, R.S, Guibal, E., Silva, E.A., \& Beppu, M.M. (2007). Adsorption and desorption of binary mixtures of copper and mercury ions on natural and crosslinked chitosan membranes. Adsorption, Vol. 13, No. 5-6, pp. 603-611. doi: 10.1007/s10450-007-9050-4

Vold, I.M.N., Vårum, K.M., Guibal, E., \& Smidsrød, O. (2003). Binding of ions to chitosan-selectivity studies. Carbohydrate Polymers, Vol. 54, No. 4, pp. 471-477. doi: $\underline{10.1016 / j . c a r b p o l .2003 .07 .001}$



Martha Benavente se graduó de Licenciada en Química en la UNAN-León, en 1987. Obtuvo el grado de MSc en Ingeniería Química en la Universidad de Chile, en 2005 y el grado de LicEng en Ingeniería Química en el Real Instituto Tecnológico (KTH), Suecia, en 2008. Sus intereses de investigación incluyen: procesamiento de biopolímeros a partir de crustáceos, remoción de metales pesados de aguas naturales y residuales. Profesor Titular, Facultad de Ingeniería Química, Universidad Nacional de Ingeniería, Nicaragua. 